\title{
The merits of ion cyclotron resonance heating schemes for sawtooth control in tokamak plasmas
}

\author{
I. T. Chapman ${ }^{1,2, \dagger}$, J. P. Graves ${ }^{1,3}$, M. Lennholm ${ }^{1,4}$, J. Faustin ${ }^{1,3}$, \\ E. Lerche ${ }^{1,5}$, T. Johnson ${ }^{1,6}$, S. Tholerus ${ }^{1,6}$ and JET contributors* \\ ${ }^{1}$ EUROfusion Consortium, JET, Culham Science Centre, Abingdon OX14 3DB, UK \\ ${ }^{2}$ CCFE, Culham Science Centre, Abingdon OX14 3DB, UK \\ ${ }^{3}$ Ecole Polytechnique Federale de Lausanne, Centre de Recherches en Physique des Plasmas, \\ 1015 Lausanne, Switzerland \\ ${ }^{4}$ European Commission, JET Exploitation Unit, Culham Science Centre, Abingdon OX14 3DB, UK \\ ${ }^{5}$ LPP-ERM/KMS, TEC Partner, Brussels, Belgium \\ ${ }^{6}$ VR, KTH, SE-100 44 Stockholm, Sweden
}

(Received 24 April 2015; revised 23 July 2015; accepted 23 July 2015)

\begin{abstract}
JET experiments have compared the efficacy of low- and high-field side ion cyclotron resonance heating (ICRH) as an actuator to deliberately minimise the sawtooth period. It is found that low-field side ICRH with low minority concentration is optimal for sawtooth control for two main reasons. Firstly, low-field side heating means that any toroidal phasing of the ICRH $\left(-90^{\circ},+90^{\circ}\right.$ or dipole $)$ has a destabilising effect on the sawteeth, meaning that dipole phasing can be employed, since this is preferable due to less plasma wall interaction from Resonant Frequency (RF) sheaths. Secondly, the resonance position of the low-field side ICRH does not have to be very accurately placed to achieve sawtooth control, relaxing the requirement for real-time control of the RF frequency. These empirical observations have been confirmed by hybrid kinetic-magnetohydrodynamic modelling, and suggest that the ICRH antenna design for ITER is well positioned to provide a control actuator capable of having a significant effect on the sawtooth behaviour.
\end{abstract}

\section{Introduction}

The baseline scenario in ITER for achieving fusion yield of $Q=10$ (Shimada 2007) - that is to say, 10 times more fusion power out than the power required to initiate plasma burn - is expected to have sawtooth oscillations in the plasma core. The sawtooth instability in the core of tokamak fusion plasmas (Levinton et al. 1993) has been observed in every tokamak and is manifest as a redistribution of the core plasma during a magnetic field reconnection event (Kadomtsev 1976; Hastie \& Hender 1988).

$\dagger$ Email address for correspondence: ian.chapman@ccfe.ac.uk

* See the appendix of Romanelli et al., Proceedings of the 25th IAEA Fusion Energy

Conference 2014, Saint Petersburg, Russia. 
Both theory (Hastie \& Hender 1988; Porcelli 1991) and experimental evidence (Park et al. 2006; Chapman et al. 2010b) suggest that this reconnection process occurs due to the growth of a plasma magnetohydrodynamic (MHD) kink instability with $m=n=1$, where $m$ and $n$ are the poloidal and toroidal periodicities, respectively. Whilst these quasi-periodic redistribution events are not anticipated to be detrimental to the performance of ITER plasmas as long as the period between reconnection events is longer than the slowing-down time of the fusion-born alpha particles, the triggering of other MHD instabilities by the sawteeth can have implications for fusion yield. It has been observed that sawteeth trigger higher- $m$ neoclassical tearing modes (NTMs) (Sauter et al. 2002), and that this triggering is exacerbated at low plasma rotation, as expected in ITER. Furthermore, the presence of fusion-born alpha particles is expected to have a stabilising influence on the internal kink mode (Porcelli, Boucher \& Rosenbluth 1996; Hu, Betti \& Manickam 2006), which leads to longer sawtooth periods, which have been empirically observed to increase the likelihood of triggering NTMs (Chapman et al. 2010a). This observation has resulted in efforts to deliberately destabilise the sawtooth oscillations, to avoid the long sawtooth periods associated with triggering NTMs at lower core pressures. An ancillary purpose for sawtooth control (which is to say the deliberate timing of the sawtooth period and ensuing determination of the amplitude of the crash) is to affect the population of impurities in the plasma core. The advent of tokamaks with tungsten divertors as planned for ITER, such as JET (Romanelli et al. 2015) or ASDEX Upgrade (Zohm et al. 2015), has highlighted the need to develop mechanisms to remove the high- $Z$ impurities that occur due to erosion of the divertor plates, as well as removing the helium ash from the plasma core, which is known to adversely affect plasma confinement (Nunes et al. 2015). The two primary mechanisms for destabilising sawteeth are either by increasing the local magnetic shear near the rational surface where sawteeth are localised, the $q=m / n=1$ surface, where $q$ is the safety factor (Porcelli et al. 1996; Eriksson et al. 2006), or by tailoring the energetic ion population to reduce the potential energy required to drive the kink mode (Graves 2005, 2009).

The sawtooth control actuators envisaged for ITER (Chapman et al. 2013a) are electron cyclotron current drive (ECCD) to affect the local magnetic shear and ion cyclotron resonance heating (ICRH) to tailor the fast-ion distribution and by so doing counteract the stabilising effects of the alpha population. The presence of fusion-born alphas in a burning D-T plasma is expected to be so stabilising to the $n=m=1 \mathrm{kink}$ mode that ICRH will be necessary to complement current drive schemes (Chapman et al. 2013a), which have been demonstrated to be particularly effective even at low power in present-day machines (Goodman et al. 2011; Igochine et al. 2011; Chapman et al. 2012, 2013b). In this paper we concentrate on the effects of tailoring the distribution of energetic particles using ICRH to control sawteeth. In recent years, ICRH sawtooth control has been demonstrated on JET using resonant frequency (RF) heating schemes with the resonance on either the low- or high-field side of the magnetic axis, as well as for various concentrations of the minority species resonant with the ion cyclotron waves from low $\left(n_{\text {min }} / n_{\text {bulk }}<10 \%\right)$ to high $\left(n_{\text {min }} / n_{\text {bulk }} \sim 30 \%\right)$ concentrations. Furthermore, the toroidal direction of propagating waves has also been changed in JET, with ensuing variations in both the radial and pitch-angle space distributions of the ICRH super-Alfvénic ions.

This paper summarises the efficacy of the different ICRH schemes for sawtooth control in JET and so infers the likely optimal solution for ITER. In $\S 2$ the mechanisms for sawtooth control are briefly recapitulated together with a brief review of the ICRH capability in ITER. The effect of the resonance position of the 
ICRH deposition on the effectiveness of sawtooth control in JET is compared to numerical modelling in $\S 3$, before the effect of the minority concentration on ICRH sawtooth control is discussed in $\S 4$. Finally, the merits of the different schemes are discussed for ITER application in $\S 5$ before conclusions are drawn.

\section{Review of sawtooth control mechanisms and ITER capability}

\subsection{Sawtooth control mechanisms}

A heuristic model for the triggering of a sawtooth crash was developed in Porcelli et al. (1996). Whilst this model does not capture all the known effects on the stability of the internal kink mode, it has had significant success in interpreting the behaviour of sawteeth in a number of tokamak plasmas (Angioni et al. 2002, 2003; Zuc 2008; Kim et al. 2014). The model consists of three different criteria, the satisfaction of which is predicted to incur a sawtooth crash. In plasmas with a large fast-ion population, the criterion that ultimately determines the crash is that

$$
s_{1}>\min \left(s_{\text {crit }}=\frac{4 \delta W}{\xi_{0}^{2} \epsilon_{1}^{2} R B^{2} c_{\rho} \hat{\rho}}, s_{c r i t}\left(\omega_{*}\right)\right) \text {. }
$$

Here the ideal growth rate normalised to the Alfvén frequency is $\gamma_{I} / \omega_{A}=-\pi \delta \hat{W} / s_{1}$, where the change in the normalised potential energy of the kink mode is $\delta \hat{W}=$ $\delta W /\left(2 \pi^{2} \xi_{r}^{2} R_{0} B_{0}^{2} \epsilon_{1}^{2}\right)$, with the change in the potential energy of the kink mode given by $\delta W=\delta W_{f}+\delta W_{K O}+\delta W_{h}$, where $\delta W_{f, K O, h}$ are the potential energy change due to the fluid drive (Bussac et al. 1975), the thermal ions (Kruskal \& Oberman 1958) and the fast ions (Graves et al. 2011), respectively. The magnetic shear at the $q=1$ surfaces is $s_{1}=\mathrm{d} q / \mathrm{d} r, R_{0}$ and $B_{0}$ are the major radius and magnetic field at the position of the magnetic axis, respectively, $\hat{\rho}$ is the normalised Larmor radius, $c_{\rho}$ is a constant of order unity, $\epsilon_{1}=r_{1} / R_{0}$ is the inverse aspect ratio, $r_{1}$ is the minor radius at the $q=1$ position, $\xi_{r}(r)$ is the leading-order rational eigenfunction of the $m=n=1$ mode at the magnetic axis and finally the critical magnetic shear determined by the pressure gradient is given by $s_{1}>s_{\text {crit }}\left(\omega_{* i}\right)$ where $\omega_{* i}$ is the ion diamagnetic frequency. The inequality condition given in (2.1) indicates that a sawtooth crash can be instigated either by increasing the local magnetic shear, $s_{1}$, or by reducing the potential energy of the kink mode, primarily through the contribution from fast particles, $\delta W_{h}$. The ICRH can modify (2.1) via either or both of these quantities since the high-energy ion populations born from RF heating can also give rise to a fairly strong local current perturbation, which when appropriately positioned can affect $s_{1}$ (Eriksson et al. 2006). Two ion cyclotron current drive (ICCD) schemes have been used, namely (i) minority ICCD, where a minority ion species resonates with the fundamental cyclotron frequency of the ICRH wave, absorbing the RF power and carrying the fast-ion current, and (ii) second harmonic ICCD, where an ion species (not necessarily a minority species) resonates at its second harmonic cyclotron frequency, $\omega=2 \omega_{c i}$ with the RF waves.

The current drive resulting from resonant minority wave-particle interactions at cyclotron frequencies, or majority ions at harmonics of the cyclotron frequency, relies on an asymmetry in the passing ion distribution induced by directed wave spectra (i.e. waves propagating preferentially in one direction) and on the velocity dependence of the collisional pitch angle scattering (Fisch et al. 1987). The resonance condition between a wave and the cyclotron motion of the resonant particles is given by $\omega-n \omega_{c i}-k_{\|} v_{\|}=0$, meaning that preferentially propagating waves can resonate 
with either co-transiting or counter-transiting ions, depending on the direction of wave propagation and the location of the interaction with respect to the cyclotron resonance. The Fisch et al. (1987) model predicts that waves propagating in the co-current direction $\left(k_{\|}>0\right)$ result in driven current with a dipole structure characterised by a positive part with respect to the plasma current on the low-field side of the cyclotron resonance and a negative part on the high-field side. For counter-propagating waves, the currents in the dipole structure change sign. This mechanism is reviewed in Bhatnagar et al. (1994) and Eriksson et al. (2006).

As well as the potential to drive current and change the local shear, the ICRH also generates very energetic particles, of course. These energetic ions directly contribute to $\delta W$ since (Porcelli 1991; Porcelli et al. 1994; Briezman et al. 1998)

$$
\delta W_{h}=\frac{1}{2} \int \mathrm{d} \Gamma\left(M v_{\|}^{2}+\mu B\right) \delta f \sum_{m} \boldsymbol{\kappa} \cdot \boldsymbol{\xi}^{(m) *}(r, t) \mathrm{e}^{-\mathrm{i}(n \phi-m \theta)},
$$

where $\theta$ is the poloidal angle, $\boldsymbol{\kappa}=\boldsymbol{b} \cdot \boldsymbol{\nabla} \boldsymbol{b}$ is the magnetic curvature vector, $\boldsymbol{b}=\boldsymbol{B} / B$, $\delta f$ is the change in the energetic particle distribution function due to the $n=m=1$ kink perturbation, $\mu$ is the magnetic moment, $\Gamma$ is phase space, $M$ is the particle mass, $v_{\|}$is the parallel velocity of the particle, $r$ is the radial position of the particle, and $\phi$ and $\theta$ are the toroidal and poloidal angles. For many years it has been known that trapped energetic particles result in strong stabilisation of sawteeth (Porcelli 1991). However, passing fast ions can also significantly influence sawtooth behaviour. For highly energetic ions, the radial drift motion becomes comparable to the radial extent of the kink mode. The strong contribution of the circulating particles comes from the ions close to the trapped-passing boundary where their orbit widths, $\Delta_{b}$, are large, $\delta W_{h}^{p} \sim \Delta_{b}$. Passing fast ions can destabilise the internal kink mode when they are co-passing and the fast-ion distribution has a positive gradient across $q=1$, or when they are counter-passing, but the deposition is peaked outside the $q=1$ surface. This mechanism is described in detail in Graves $(2004,2009)$ with an overview of fast-ion effects in Chapman et al. (2011a).

Recent experiments in JET have isolated the dominant mechanism for sawtooth control with ICRH as the effect from energetic ions (Graves et al. 2012, 2015). By using ${ }^{3} \mathrm{He}$-minority heating, the ion cyclotron-driven current is negligible due to the current dragged by the background plasma, which tends to cancel the ${ }^{3} \mathrm{He}$ current (Laxåback \& Hellsten 2005), but yet the control of sawteeth is still highly efficient (Graves et al. 2012). The dominance of the fast-ion contribution was unequivocally demonstrated by variation of the minority concentration. At very low concentration, the absorbed power is reduced, and consequently the effect on the sawteeth was mild. Similarly, at high concentration, the energy tail of the energetic ion population is lower, so the radial drift excursion of the ICRH fast ions which intersect the $q=1$ surface is smaller, and so the ability to affect the sawteeth is again reduced. However, at minority concentrations intermediate between these extremes, the radial orbit drift of the energetic ion distribution tail and the absorbed power are both sufficient to have a strong effect on the sawteeth, even in H-mode in the presence of a population of stabilising core fast ions when there is negligible drive current. Furthermore, experiments with low-field side H-minority ICRH showed that any phasing of the ICRH $\left(-90^{\circ},+90^{\circ}\right.$ or dipole) has a destabilising effect on the sawteeth, i.e. reduces the sawtooth period, when the resonance position is just outside the $q=1$ surface. Whilst the driven current is mild, in all cases the magnetic shear at $q=1$ is actually reduced, which should stabilise the sawteeth, whilst a marked 
destabilisation is observed, emphasising that the conventional 'change in magnetic shear' interpretation for the control of sawteeth cannot apply in these H-minority low-field side experiments, and furthermore that the fast-ion effects on the kink mode could be inferred to be typically the dominant effect of ICRH.

\subsection{ITER capabilities}

ITER is expected to have $20 \mathrm{MW}$ of ICRH as part of the portfolio of $73 \mathrm{MW}$ of auxiliary heating and current drive tools. Ion cyclotron waves will be strongly damped in ITER, leading to various possible heating schemes: second harmonic tritium or fundamental ${ }^{3} \mathrm{He}$ heating; fundamental $\mathrm{D}$ heating (although a low deuterium concentration is required for efficient wave damping, making this low relevance); electron Landau damping (ELD)/transit time magnetic pumping (TTMP), both of which are fast wave current drive scenarios leading to insignificant fast-ion populations; or off-axis ${ }^{3} \mathrm{He}$ heating, which has moderate absorbed power density but can result in a significant population of ICRH energetic ions near the $q=1$ surface. At full magnetic field in ITER $\left(B_{0}=5.3 \mathrm{~T}\right)$, the ICRH antenna design precludes high-field side resonance positions. Variable-frequency ICRH is currently planned for ITER. The ICRH frequency will be able to vary in real time between 40 and $55 \mathrm{MHz}$ (Lamalle et al. 2013). The ${ }^{3} \mathrm{He}$ resonance just outside $q=1$ is likely to require a frequency of approximately $47 \mathrm{MHz}$. It will be possible to rapidly change the RF frequency in a preset $2 \mathrm{MHz}$ band, which is equivalent to moving the resonance location by approximately $20 \mathrm{~cm}$, which should provide sufficient scope for real-time sawtooth control. In the ${ }^{3} \mathrm{He}$-minority heating scheme envisaged for ITER, the resultant current drive is negligible (Laxåback \& Hellsten 2005; Graves 2009).

\section{The effect of the ICRH resonance position on sawtooth control in JET}

The ICRH system in JET has a wide frequency variation, meaning that resonance positions on either the low- or high-field side of the magnetic axis are possible. In this section we describe the merits of high-field side heating with either $\mathrm{H}-$ or ${ }^{3} \mathrm{He}$ minority species compared to low-field side heating with $\mathrm{H}$ minority. Figure 1 shows the configuration of typical JET plasmas which have the ICRH resonance on the lowfield side (37 MHz with $B_{t} \in[2.7,2.35] \mathrm{T}$ using fundamental $\mathrm{H}$-minority resonance) and on the high-field side (33 MHz with $B_{t} \in[2.88,2.96] \mathrm{T}$ using fundamental ${ }^{3} \mathrm{He}$ minority resonance) compared to the approximate position of the $q=1$ surface found from the sawtooth inversion radius measured by soft X-ray emission in JET discharge 84500 , which is typical of the plasmas used in this study. In both cases an optimal minority concentration is employed.

\subsection{Sawtooth control with low-concentration ${ }^{3} \mathrm{He}$-minority ICRH resonance on the high-field side}

Sawtooth control was demonstrated with ${ }^{3} \mathrm{He}$-minority heating on the high-field side, whereby the current drive was minimised to verify that the passing fast-ion effect dominated (Graves et al. 2010, 2012). In order to demonstrate the effect of the ICRH fast ions on the sawtooth behaviour, the ICRH resonance position has been moved with respect to the $q=1$ surface by ramping the magnetic field to move the ICRH resonance and the current commensurately to keep the $q$ profile fixed. Typically the ICRH resonance is moved from outside the $q=1$ surface, where the ICRH barely affects the sawtooth behaviour, to inside it, where it is known that the 


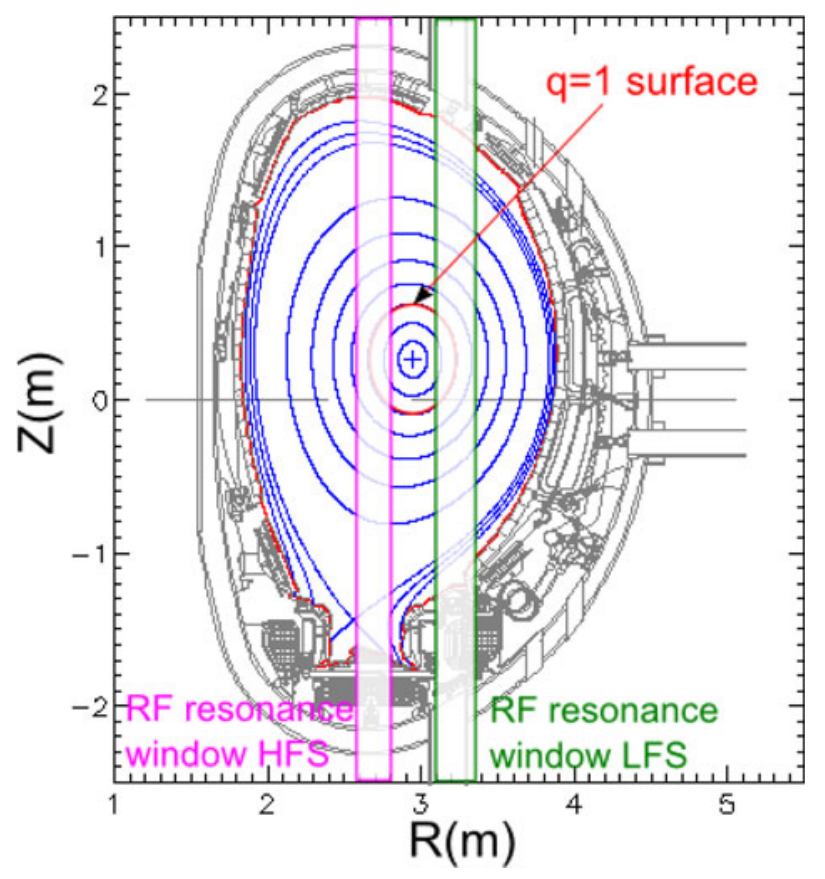

FIgURE 1. The plasma configuration used in JET discharge 84500 at $15 \mathrm{~s}$, which is representative of the plasmas studied in this paper, showing the $q=1$ surface and the ICRH resonances for ${ }^{3} \mathrm{He}$ on the high-field side and $\mathrm{H}$ on the low-field side.

ICRH trapped energetic particles have a strongly stabilising effect, increasing the sawtooth period. Through sweeps like this, the change in the sawtooth period when the resonance position is just in/outside $q=1$ can be studied, since it is at this point that the fast-ion population with radial drift excursions cutting the rational surface is maximised, and it is this population deliberately placed near the $q=1$ surface that affects kink stability (Graves 2009). Figure 2 shows the sawtooth behaviour as the ICRH resonance is swept from outside to inside the $q=1$ surface when the resonance is on the high-field side of the magnetic axis. It is evident that the sawtooth period is minimised as the RF resonance is positioned just inside the $q=1$ surface. Note that this occurs for $r_{r e s}<r_{i n v}$ even though $R_{r e s}>R_{i n v}$ when the resonance is on the high-field side. For effective sawtooth destabilisation, i.e. a minimisation of the sawtooth period, the location of the RF resonance must be highly accurately placed with respect to the rational surface, with a localisation closer than $\sim 2 \mathrm{~cm}$. Given significant uncertainty in the radial localisation of the $q=1$ surface in ITER, and the ray tracing to predict the resonance position being subject to uncertainties in the plasma density profile as well as approximations in the ray propagation models, this narrow window for effective sawtooth control implies that a real-time feedback scheme is needed for robust high-field side ICRH sawtooth control (Lennholm et al. 2011).

It is found that the toroidal phasing of the ICRH antenna configuration has a marked affect on the sawtooth control for high-field side resonance (Graves et al. 2010). When the ICRH has $-90^{\circ}$ phasing, the ICRH resonance just inside the $q=1$ surface gives rise to destabilisation of the kink mode, and so shortening of the sawtooth period, whilst $+90^{\circ}$ phasing has the opposite effect, stabilising the sawteeth. 
(a)

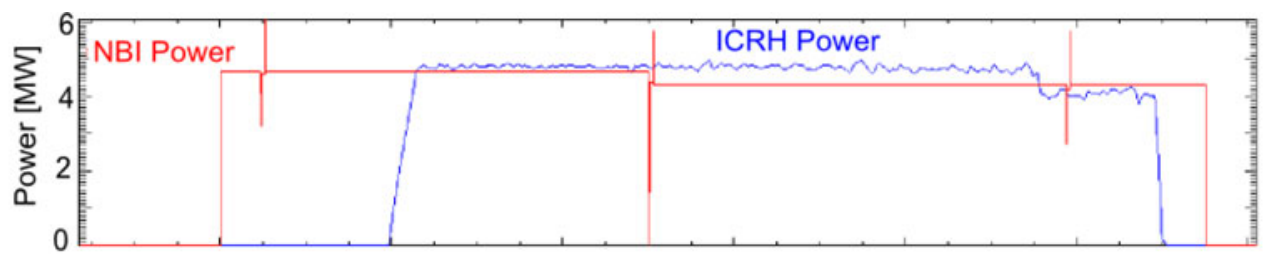

(b)

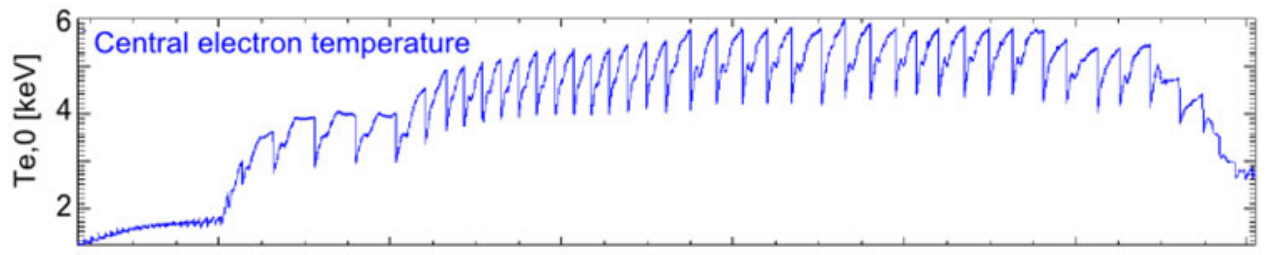

(c)
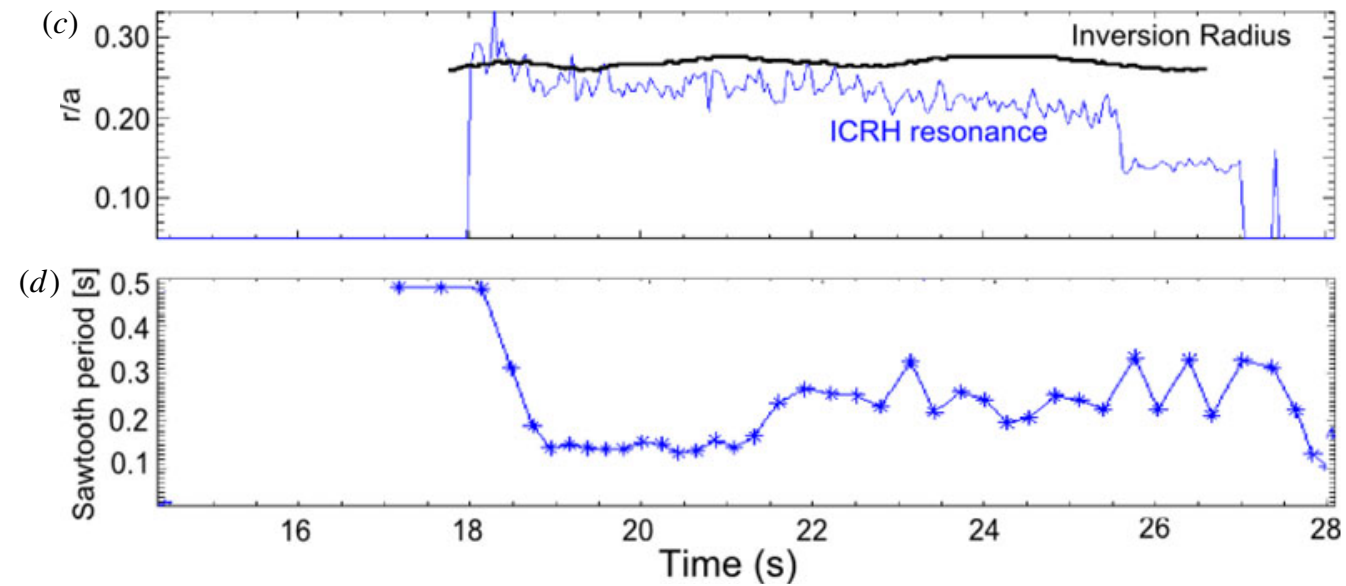

FIGURE 2. Time traces for JET pulse 78764 showing (a) the auxiliary NBI and ICRH heating power, $(b)$ the central electron temperature measured by the electron cyclotron emission diagnostic demonstrating the sawtooth activity, $(c)$ the resonance position of the high-field side ICRH compared to the radial position of the sawtooth inversion radius, and (d) the sawtooth period. The ICRH had $-90^{\circ}$ phasing. The toroidal magnetic field was ramped linearly from 2.85 to $2.95 \mathrm{~T}$ and proportionately the plasma current was ramped from 1.95 to 2.05 MA.

This is illustrated in figure 3, which shows the sawtooth period as a function of the resonance position with respect to the $q=1$ surface for both $\pm 90^{\circ}$ phasings when the ICRH resonance is on the high-field side. It is clear that when the resonance is just inside $q=1$ the counter-propagating waves give rise to a minimum in the achievable sawtooth period, significantly lower than the reference case without ICRH, whilst the co-propagating waves result in a significant increase in the sawtooth period. In this case, the lengthening of the sawtooth period to $\sim 1 \mathrm{~s}$ actually results in the triggering of an NTM, even in L-mode at $\beta_{N}=0.8$ (well below the target level of the $Q=10$ scenario for ITER, where $\beta_{N}=1.8$ ). At this point it is worth pointing out that phased ICRH waves are considered sub-optimal compared to dipole phasing because of the slightly lower absorbed power and, importantly, the enhanced plasma-wall interaction due to RF sheath redistribution effects (Czarnecka et al. 2011), which is a considerable limitation in devices with metal walls owing to the sputtering of tungsten. 


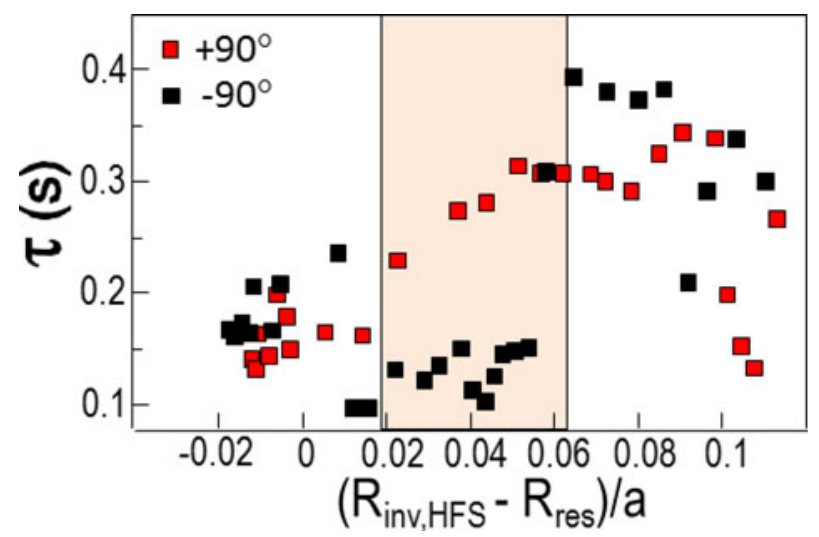

FIGURE 3. The sawtooth period as a function of the resonance position with respect to the inversion radius for different ICRH phasings in JET for ICRH with low $\left(\sim 0.5 \%{ }^{3} \mathrm{He}\right)$-minority concentration located on the high-field side. The shaded area represents the region with an ICRH resonance just outside the $q=1$ surface, where the fast ions have the strongest effect on the sawtooth behaviour.

In order to understand the effect of these different fast-ion distributions on the stability of the internal kink mode, coupled hybrid kinetic-MHD numerical modelling has been employed. The ICRH fast-ion population is modelled with either the SELFO (Hedin et al. 2002) or SCENIC (Jucker et al. 2011) ICRH wave codes, whilst the neutral beam injection (NBI) distribution is modelled with the NUBEAM model within the TRANSP code (Budny et al. 1992). Meanwhile, the plasma equilibrium is constrained by the sawtooth inversion radius and reconstructed using the HELENA (Huy 1991) Grad-Shafranov solver. The linear MHD stability of this equilibrium is then studied using the MISHKA code (Chapman et al. 2006) to find an unstable $n=m=1$ eigenfunction. The interaction between this unstable kink mode and the fast ions is then studied with the drift-kinetic HAGIS code (Pinches et al. 1998), which calculates $\delta W_{h}$. Figure 4 shows the change in the potential energy of the kink mode as a function of radial position of the peak of the ICRH distribution with respect to the $q=1$ surface position. It is evident that the $-90^{\circ}$ phasing reduces $\delta W_{h}$, consistent with the reduction in the sawtooth period observed in JET, whilst the $+90^{\circ}$ phasing increases $\delta W_{h}$, once again consistent with the experimental sawtooth behaviour. The effect on kink stability is governed by the asymmetry in the parallel velocity of the ICRH distribution function, and specifically in the radial variation of this asymmetry. When the population of fast ions is such that the number of co- and counter-passing ICRH ions whose radial drift is large enough that it cuts across the $q=1$ surface is unequal, they result in a change in the kink mode potential (Graves 2009; Chapman et al. 2011a).

\subsection{Sawtooth control with low-concentration H-minority ICRH resonance on the low-field side}

Similar sweeps of the ICRH resonance position across the $q=1$ surface have been performed with the resonance located on the low-field side of the magnetic axis in JET, as expected for ITER at full magnetic field. In JET this means using H-minority ICRH heating, which does drive a small ion cyclotron current, but, as demonstrated 


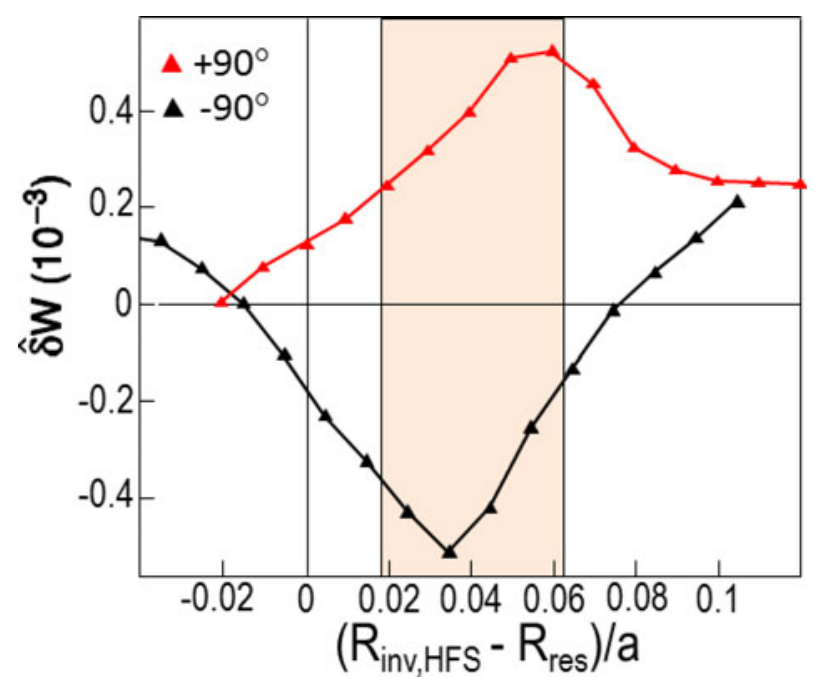

FIGURE 4. The $\delta \hat{W}_{h}$ calculated by HAGIS for different ICRH phasings with the ICRH resonance on the high-field side and the ICRH distribution taken from SELFO modelling. The shaded band is the same as in figure 3, demonstrating that for $-90^{\circ}$ phasing the $\delta \hat{W}_{h}$ is negative, i.e. destabilising in this region, in line with the shorter sawtooth period seen in figure 3. Conversely, the $+90^{\circ}$ phasing in the same region gives positive $\delta \hat{W}_{h}$, i.e. sawtooth stabilisation, commensurate with the longer sawtooth period observed experimentally.

in Graves et al. (2015), the effect on the sawteeth is dominated by the fast-ion mechanisms over the shear mechanism. Figure 5 shows the sawtooth behaviour as the ICRH resonance is swept from outside to inside the $q=1$ surface when the resonance is on the low-field side of the magnetic axis. It is evident that the sawtooth period is minimised as the RF resonance is positioned close to the $q=1$ surface. However, in contrast to the result with high-field side resonance, the window for effective sawtooth destabilisation, i.e. a reduction of the sawtooth period, is significantly wider. In the case of low-field side resonance, the resonance position must be in a window of only $\sim 15 \mathrm{~cm}$ around the $q=1$ surface to achieve sawtooth destabilisation, which is much broader than for high-field side ICRH. If this can be replicated in plasma with a dominant core fast-ion population, this would negate the requirement for real-time control of the RF frequency, greatly simplifying the coupling of the RF waves to the plasma.

The toroidal phasing of low-field side RF schemes has also been varied in order to compare to the result from high-field side heating, where the sawtooth control was strongly dependent upon the ICRH phasing. Figure 6 shows the sawtooth period as a function of the resonance position with respect to the $q=1$ surface for $-90^{\circ},+90^{\circ}$ and dipole phasings when the ICRH resonance is on the low-field side. In contrast to high-field side ICRH sawtooth control, all phasings have a destabilising effect on the sawteeth when the resonance is on the low-field side. Again, this is positive for ITER if it can be sustained in plasmas with a high fast-ion fraction, since using dipole phasing does not have the by-product of enhanced plasma-wall interaction.

Finally, the change in the kink mode stability resulting from the low-field side ICRH fast-ion populations has also been modelled using the hybrid MHD-kinetic code package as before. Figure 7 shows the change in the potential energy of the 


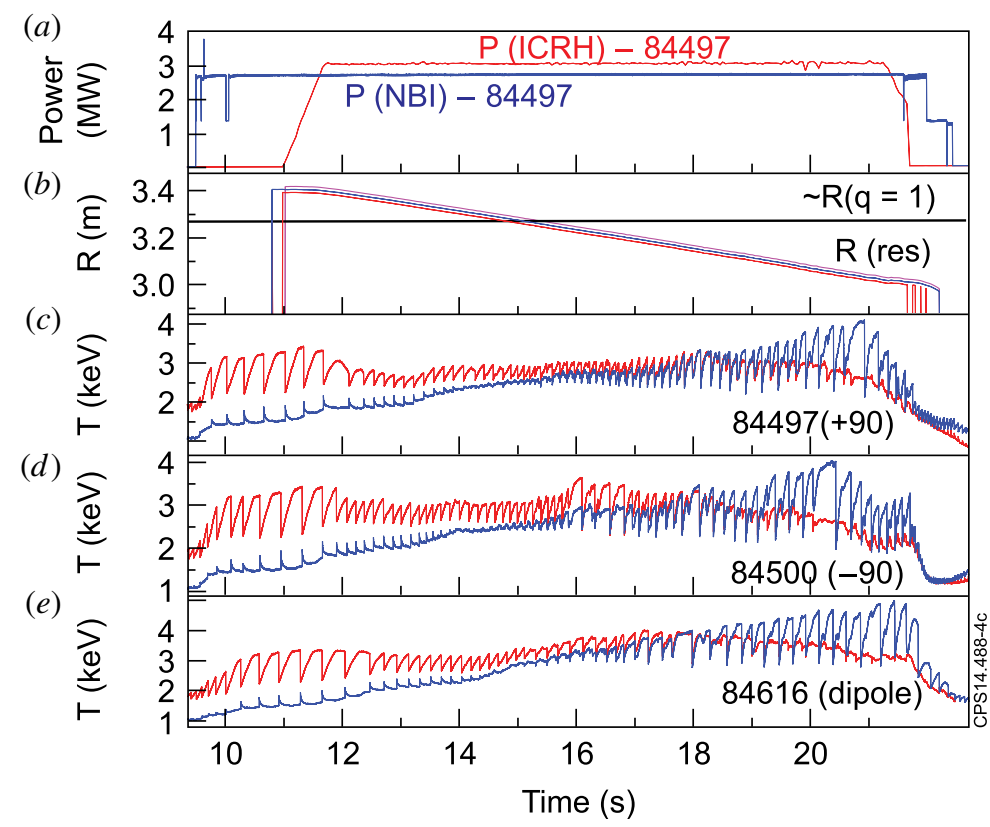

FIGURE 5. Time traces for three similar JET pulses with different ICRH phasings showing (a) the auxiliary NBI and ICRH heating power, $(b)$ the resonance position of the low-field side ICRH compared to the radial position of the sawtooth inversion radius and $(c-e)$ the central electron temperature measured by the electron cyclotron emission diagnostic, demonstrating the sawtooth activity for $+90^{\circ},-90^{\circ}$ and dipole ICRH phasing, respectively. The toroidal magnetic field was ramped linearly from 2.7 to $2.35 \mathrm{~T}$ and proportionately the plasma current was ramped from 2.4 to $2.05 \mathrm{MA}$.

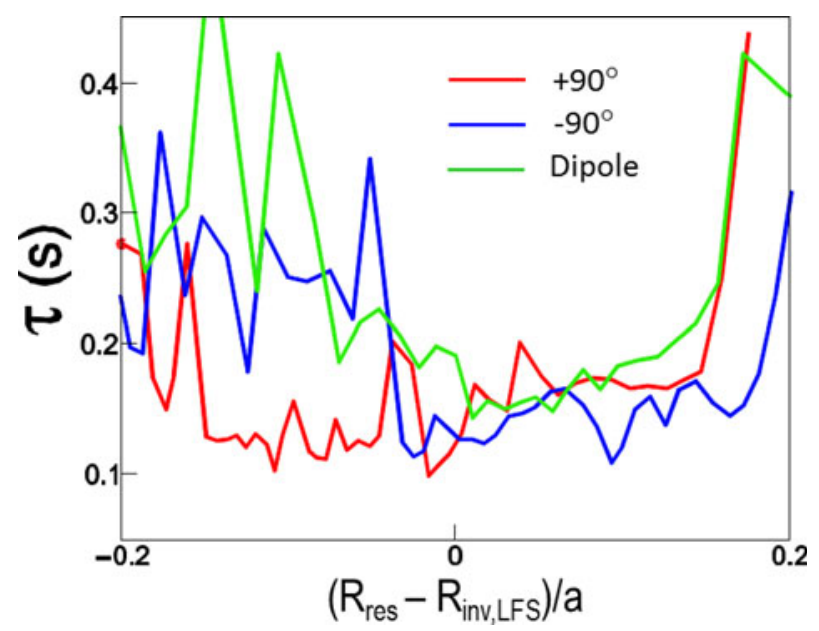

FIGURE 6. The sawtooth period as a function of the resonance position with respect to the inversion radius for different ICRH phasings in JET for ICRH with $\sim 4 \% \mathrm{H}$-minority concentration located on the low-field side. 


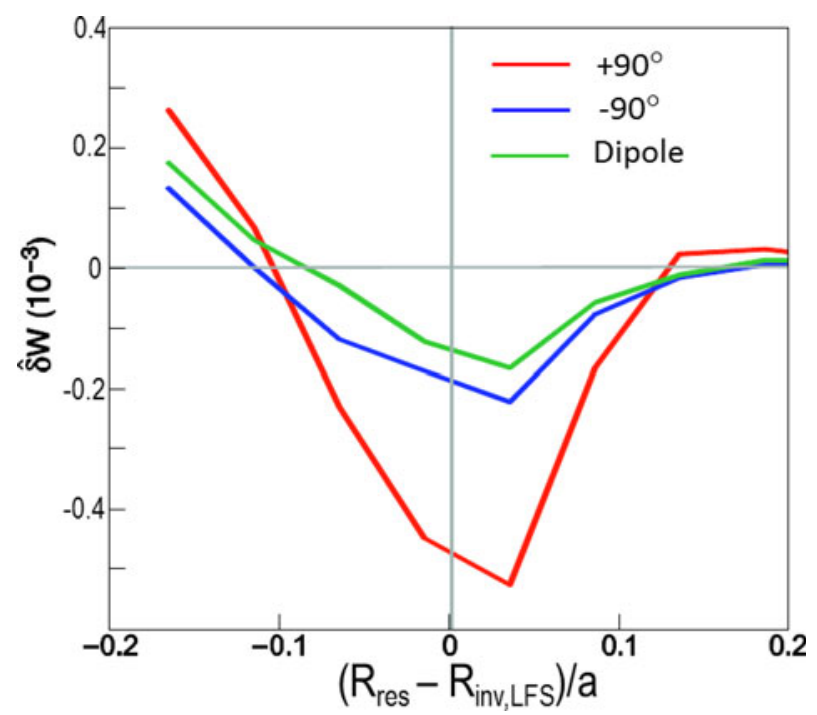

FIGURE 7. The $\delta \hat{W}_{h}$ calculated by HAGIS for different ICRH phasings with the ICRH resonance on the low-field side and the ICRH distribution taken from SCENIC modelling.

kink mode as a function of the radial position of the peak of the ICRH distribution with respect to the $q=1$ surface position. Whilst the $+90^{\circ}$ ICRH population has the most destabilising effect on the kink mode, as expected from Graves (2009), all of the phasings do reduce $\delta W_{h}$. Qualitatively these simulations verify the empirical observations, namely that with the ICRH resonance on the low-field side the $+90^{\circ}$ phasing is most destabilising, but that all phasings do reduce kink stability provided the resonance is centred just outside $q=1$. Furthermore, the window in $r_{r e s}-r_{1}$ for which $\delta W_{h}$ is reduced is much broader for low-field side heating than when the resonance is on the high-field side.

\section{The effect of the minority concentration on sawtooth control}

It was shown in Graves et al. (2012) that sawtooth destabilisation from high-field side ICRH was extremely sensitive to the minority species concentration. This is essentially due to the fast-ion (de)stabilisation mechanism dependence on the need to develop an asymmetry in the parallel velocity of the passing fast ions that cross the $q=1$ surface. If the minority concentration is too high, there is an insufficient number of fast ions that have sufficient energy to have a broad radial drift excursion and so cut across $q=1$. Equally, if the minority concentration is too low, whilst there is a long tail in the energy distribution with a large fraction of very energetic particles with large effective orbit width, the total absorbed power is too low to compete with the stabilising NBI fast ions. This dependence on the minority concentration is demonstrated in figure 8 , where the $\delta W_{h}$ modelled by HAGIS is shown for ICRH with a resonance position located on the high-field side with $-90^{\circ}$ antenna phasing as the resonance is swept from outside to inside the $q=1$ surface. Strong destabilisation is only observed for intermediate minority concentration $\left(n_{3 \mathrm{He}} / n_{\text {bulk }} \sim 1 \%\right)$, whilst a weak effect on the sawteeth is seen for both low $\left(n_{3 \mathrm{He}} / n_{\text {bulk }} \sim 0.15 \%\right)$ and high $\left(n_{\text {3 }} / n_{\text {bulk }} \sim 3 \%\right)$ concentrations. 


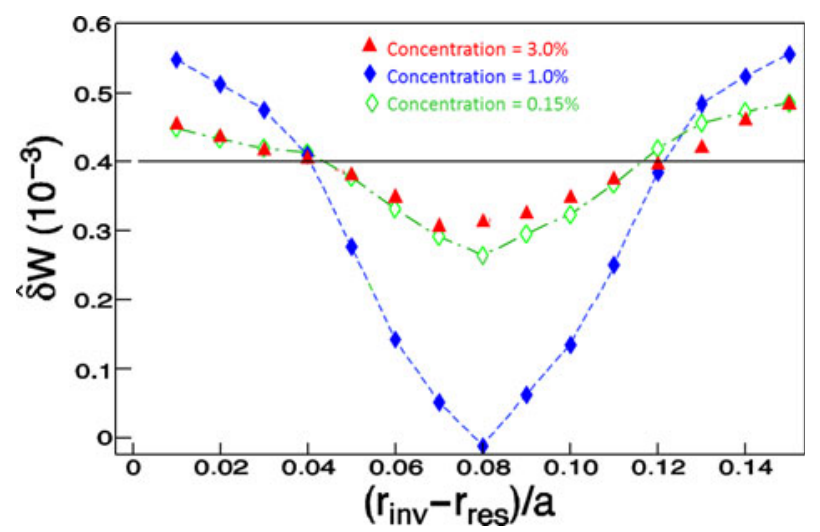

FIgURE 8 . The $\delta \hat{W}_{h}$ calculated by HAGIS as a function of the resonance position with respect to the sawtooth inversion radius in JET plasmas when the ICRH resonance is on the high-field side for different ${ }^{3} \mathrm{He}$-minority concentrations.

\section{Discussion of the merits of ICRH control schemes in ITER and conclusions}

In $\S 3$ it is shown that low-field side resonance heating has a significantly broader window in the resonance position to destabilise the sawteeth. With high-field side ICRH, the resonance must be placed within $\sim 2 \mathrm{~cm}$ of the $q=1$ surface in JET in order to reduce the sawtooth period. Conversely, with low-field side ICRH, this window is increased to $\sim 15 \mathrm{~cm}$, greatly reducing the likely need for real-time control of the resonance position through changing the RF frequency (Lennholm et al. 2015). Furthermore, heating with a resonance on the low-field side is effective no matter which phasing of the ICRH antenna is employed. Even ICRH waves injected perpendicular to the magnetic field can destabilise the sawteeth, whereas dipole phasing is always observed to be stabilising on the high-field side. In $\S 4$ it is demonstrated that by simultaneously optimising the coupled RF power and the energy of the ICRH distribution tail such that the radial width of the orbit excursion of particles crossing the $q=1$ surface is maximised, the effect on the sawteeth is optimised. This occurs for intermediate minority concentrations $\left(n_{\text {min }} / n_{\text {bulk }} \sim 3-6 \%\right)$ in JET for $\mathrm{H}$ minority, but at lower concentration $\left(n_{\min } / n_{\text {bulk }} \sim 0.5-1 \%\right)$ for ${ }^{3} \mathrm{He}$ minority.

The anticipated ICRH heating scheme in ITER uses $51 \mathrm{MHz}$ RF heating of a low-concentration ${ }^{3} \mathrm{He}$-minority species on the low-field side of the plasma. The results from JET coupled with the numerical modelling in $\S \S 3$ and 4 suggest that this is the optimal ICRH antenna design for sawtooth control, for two reasons. (i) Low-field side heating means that any toroidal phasing of the ICRH $\left(90^{\circ},+90^{\circ}\right.$ or dipole) has a destabilising effect on the sawteeth, i.e. reduces the sawtooth period, meaning that dipole phasing can be employed, since this is preferable due to less plasma-wall interaction from RF sheaths (Czarnecka et al. 2011) with a metal wall. (ii) The resonance position of the low-field side ICRH does not have to be very accurately placed to achieve sawtooth control. This relaxes the necessity for real-time control of the RF frequency. Calculations of the change of the kink mode potential energy due to ICRH fast ions using the SCENIC and HAGIS codes, as described in $\$ 3$, have been carried out for low-field side ICRH heating in ITER using ${ }^{3} \mathrm{He}$ minority with different antenna phasings and for different minority concentrations (Chapman et al. 2011b). It is found that the most destabilising effect on the internal kink mode is achieved for $n^{3 \mathrm{He}} / n_{\text {bulk }} \sim 1 \%$ and, whilst $+90^{\circ}$ phasing is optimal, 
a strong effect is attained even with dipole phasing. Together with the empirical observations from JET, this numerical modelling suggests that the ICRH antenna design for ITER is well positioned to provide a control actuator capable of having a significant effect on the sawtooth behaviour, and, coupled with the electron cyclotron current drive, can keep the sawtooth period below that expected to trigger NTMs (Chapman et al. 2011b).

\section{Acknowledgements}

This work has been carried out within the framework of the EUROfusion Consortium and has received funding from the Euratom research and training programme 2014-2018 under grant agreement no. 633053 and from the RCUK Energy Programme (grant no. EP/I501045). To obtain further information on the data and models underlying this paper, please contact PublicationsManager@ccfe.ac.uk. The views and opinions expressed herein do not necessarily reflect those of the European Commission.

\section{REFERENCES}

Angioni, C., Goodman, T., Henderson, M. \& Sauter, O. 2003 Effects of localized electron heating and current drive on the sawtooth period. Nucl. Fusion 43, 455.

Angioni, C., Pochelon, A., Gorelenkov, N. N., McClements, K. G., Sauter, O., Budny, R. V., de Vries, P. C., Howell, D. F., Mantsinen, M., Nave, M. F. F., Sharapov, S. E. \& contributors to the EFDA-JET Workprogramme 2002 Neutral beam stabilization of sawtooth oscillations in JET. Plasma Phys. Control. Fusion 44, 205.

Bhatnagar, V. P., Start, D. F. H, Jacquinot, J., Chaland, F., Cherubini, A. \& Porcelli, F. 1994 Local magnetic shear control in a tokamak via fast wave minority ion current drive: theory and experiments in JET. Nucl. Fusion 34, 1579.

Briezman, B., CAndy, J., Porcelli, F. \& Berk, H. 1998 On the theory of internal kink oscillations. Phys. Plasmas 5, 2326.

Budny, R. V., Bell, M. G., Biglari, H., Bitter, M., Bush, C. E., Cheng, C. Z., Fredrickson, E. D., Grek, B., Hill, K. W., Hsuan, H., Janos, A. C., Jassby, D. L., Johnson, D. W., Johnson, L. C., LeBlanc, B., McCune, D. C., Mikkelsen, D. R., Park, H. K., Ramsey, A. T., Sabbagh, S. A., Scott, S. D., Schivell, J. F., Strachan, J. D., Stratton, B. C., Synakowski, E. J., Taylor, G., Zarnstorff, M. C. \& ZWEBEN, S. J. 1992 Simulations of deuterium-tritium experiments in TFTR. Nucl. Fusion 32, 429.

Bussac, M. N., Pellat, R., Edery, D. \& Soule, J. L. 1975 Internal kink modes in toroidal plasmas with circular cross sections. Phys. Rev. Lett. 35, 1638.

Chapman, I. T., Sharapov, S. E., Huysmans, G. T. A.\& Mikhailovskit, A. B. 2006 Modeling the effect of toroidal plasma rotation on drift-magnetohydrodynamic modes in tokamaks. Phys. Plasmas 13, 062511.

Chapman, I. T., Buttery, R. J., Coda, S., Gerhardt, S., Graves, J. P., Howell, D. F., Isayama, A., La Haye, R. J., Liu, Y., Maget, P., Maraschek, M., Sabbagh, S., SAUTER, O. \& the ASDEX Upgrade, DIII-D, HL-2A, JT-60U, MAST, NSTX, TCV and Tore Supra Teams and JET-EFDA Contributors $2010 a$ Empirical scaling of sawtooth period for onset of neoclassical tearing modes. Nucl. Fusion 50, 102001.

Chapman, I. T., Scannell, R., Cooper, W. A., Graves, J. P., Hastie, R. J., Naylor, G. \& Zocco, A. $2010 b$ Magnetic reconnection triggering magnetohydrodynamic instabilities during a sawtooth crash in a tokamak plasma. Phys. Rev. Lett. 105, 255002.

Chapman, I. T. $2011 a$ Controlling sawtooth oscillations in tokamak plasmas. Plasma Phys. Control. Fusion 53, 013001. 
Chapman, I. T., Graves, J. P., Johnson, T., Asunta, O., Bonoli, P., Choi, M., Jaeger, E. F., JUCKER, M. \& SAUTER, O. $2011 b$ Sawtooth control in ITER using ion cyclotron resonance heating. Plasma Phys. Control. Fusion 53, 124003.

Chapman, I. T., La Haye, R. J., Buttery, R. J., Heidbrink, W. W., Jackson, G. L., Muscatello, C. M., Petty, C. C., Pinsker, R. I., Tobias, B. J. \& Turco, F. 2012 Sawtooth control using electron cyclotron current drive in ITER demonstration plasmas in DIII-D. Nucl. Fusion 52, 063006.

Chapman, I. T., Graves, J. P., Sauter, O., Zucca, C., Asunta, O., Buttery, R. J., Coda, S., Goodman, T., Igochine, V., Johnson, T., Jucker, M., La Haye, R. J., Lennholm, M. \& JET-EFDA Contributors $2013 a$ Power requirements for electron cyclotron current drive and ion cyclotron resonance heating for sawtooth control in ITER. Nucl. Fusion 53, 066001.

Chapman, I. T., Igochine, V., Maraschek, M., McCarthy, P. J., Tardini, G. \& the ASDEX Upgrade ECRH Group and the ASDEX Upgrade Team 2013b Sawtooth control using electron cyclotron current drive in the presence of energetic particles in high performance ASDEX Upgrade plasmas. Plasma Phys. Control. Fusion 55, 065009.

Czarnecka, A., Zastrow, K.-D., Rzadkiewicz, J., Coffey, I. H., Lawson, K. D., O’Mullane, M. G. \& JET-EFDA Contributors 2011 Determination of metal impurity density, $\Delta Z_{\text {eff }}$ and dilution on JET by VUV emission spectroscopy. Plasma Phys. Control. Fusion 53, 035009 .

Eriksson, L.-G., Johnson, T., Mayoral, M.-L., Coda, S., Sauter, O., Buttery, R. J., McDonald, D., Hellsten, T., Mantsinen, M. J., Mueck, A., Noterdaeme, J.-M., SAntAla, M., Westerhof, E., DE VRIEs, P. \& JET-EFDA contributors 2006 On ion cyclotron current drive for sawtooth control. Nucl. Fusion 46, S951.

Fisch, N. J. 1987 Theory of current drive in plasmas. Rev. Mod. Phys. 59, 175.

Goodman, T. P., Felici, F., Sauter, O. \& Graves, J. P. 2011 Sawtooth pacing by real-time auxiliary power control in a tokamak plasma. Phys. Rev. Lett. 106, 245002.

Graves, J. P. 2004 Influence of asymmetric energetic ion distributions on sawtooth stabilization. Phys. Rev. Lett. 92, 185003.

Graves, J. P. 2005 Sawtooth control in fusion plasmas. Phys. Plasmas 12, 090908.

GRAVES, J. P. 2009 Sawtooth-control mechanism using toroidally propagating ion-cyclotron-resonance waves in tokamaks. Phys. Rev. Lett. 102, 065005.

Graves, J. P., Chapman, I. T., Coda, S., Johnson, T., Lennholm, M., Alper, B., De BaAr, M., Crombe, K., Eriksson, L.-G., Felton, R., Howell, D., Kiptily, V., Koslowski, H. R., Mayoral, M.-L., Monakhov, I., Nunes, I., Pinches, S. D. \& JeT-EFDA Contributors 2010 Experimental verification of sawtooth control by energetic particles in ion cyclotron resonance heated jet tokamak plasmas. Nucl. Fusion 50, 052002.

Graves, J. P., Chapman, I. T., Coda, S., Johnson, T., Lennholm, M., Paley, J. I., Sauter, O. \& JET-EFDA Contributors 2011 Advances in sawtooth control. Fusion Sci. Technol. 59, 539.

Graves, J. P., Chapman, I. T., Coda, S., Lennholm, M., Albergante, M. \& Jucker, M. 2012 Control of magnetohydrodynamic stability by phase space engineering of energetic ions in tokamak plasmas. Nat. Commun. 3, 624.

Graves, J. P., Lennholm, M., Chapman, I. T., Lerche, E., Reich, M., Alper, B., Bobkov, V., Dumont, R., Faustin, J. M., Jacquet, P., Jaulmes, F., Johnson, T., Keeling, D. L., Liu, Yueqiang, Nicolas, T., Tholerus, S., Blackman, T., Carvalho, I. S., Coelho, R., Van Eester, D., Felton, R., Goniche, M., Kiptily, V., Monakhov, I., Nave, M. F. F., Perez von Thun, C., Sabot, R., Sozzi, C. \& Tsalas, M. 2015 Sawtooth control in jet with ITER relevant low field side resonance ion cyclotron resonance heating and ITER-like wall. Plasma Phys. Control. Fusion 57, 014033.

Hastie, R. J. \& Hender, T. C. 1988 Toroidal internal kink stability in tokamaks with ultra flat $q$ profiles. Nucl. Fusion 28, 585.

Hedin, J., Hellsten, T., Eriksson, L.-G. \& Johnson, T. 2002 The influence of finite drift orbit width on ICRF heating in toroidal plasmas. Nucl. Fusion 42, 527. 
Hu, B., Betti, R. \& Manickam, J. 2006 Kinetic stability of the internal kink mode in ITER. Phys. Plasmas 13, 112505.

Huysmans, G. T. A. 1991 Proceedings of the CP90 Conference on Computational Physics, p. 371. World Scientific Publishing Co..

Igochine, V. G., Chapman, I. T., Bobkov, V., Günter, S., Maraschek, M., Moseev, D., Pereversev, G., Reich, M., Stober, J. \& ASDEX Upgrade team 2011 Destabilization of fast particle stabilized sawteeth in ASDEX Upgrade with electron cyclotron current drive. Plasma Phys. Control. Fusion 53, 022002.

Jucker, M., Graves, J. P., CoOper, W. A. \& Johnson, T. 2011 Ion cyclotron resonance heating with consistent finite orbit widths and anisotropic equilibria. Plasma Phys. Control. Fusion 53, 054010 .

Kadomtsev, B. B. 1976 Disruptive instability in tokamaks. Sov. J. Plasma Phys. 1, 389.

KiM, D. \& SAUTER, O. 2014 Real-time sawtooth control and neoclassical tearing mode preemption in ITER. Phys. Plasmas 21, 061503.

Kruskal, M. \& Oberman, C. 1958 On the stability of plasma in static equilibrium. Phys. Fluids 1, 275.

Lamalle, P., Beaumont, B., Kazarian, F., Gassmann, T., Agarici, G., Ajesh, P., Alonzo, T., Arambhadiya, B., Argouarch, A., Bamber, R., Berger-By, G., Bernard, J.-M., Brun, C., Carpentier, S., Clairet, F., Colas, L., Courtois, X., Davis, A., Dechelle, C., Doceul, L. \& Dumortier, P. 2013 Status of the ITER ion cyclotron H and CD system. Fusion Engng Des. 88, 517.

LaXÅBACK, M. \& Hellsten, T. 2005 Modelling of minority ion cyclotron current drive during the activated phase of ITER. Nucl. Fusion 45, 1510.

Lennholm, M., Blackman, T., Chapman, I. T., Eriksson, L.-G., Graves, J. P., Howell, D. F., de BaAr, M., Calabro, G., Dumont, R., Graham, M., Jachmich, S., Mayoral, M. L., Sozzi, C., Stamp, M., Tsalas, M., de VRies, P. \& JeT EFDA Contributors 2011 Feedback control of the sawtooth period through real time control of the ion cyclotron resonance frequency. Nucl. Fusion 51, 073032.

Lennholm, M., Frigione, D., Graves, J., Beaumont, P. S., Blackman, T., Carvalho, I. S., Chapman, I., Dumont, R., Felton, R., Garzotti, L., Goniche, M., Goodyear, A., Grist, D., Jachmich, S., Johnson, T., Lang, P., Lerche, E., DE la Luna, E., Monakhov, I., Mooney, R., Morris, J., Nave, M. F. F., Reich, M., Rimini, F., Sips, G., Sheikh, H., Sozzi, C., Tsalas, M. \& JET contributors 2015 Real-time control of ELM and sawtooth frequencies: similarities and differences. Nucl. Fusion (submitted).

Levinton, F. M., Batha, S. H., Yamada, M.\& ZarnstorfF, M. C. 1993 q-profile measurements in the tokamak fusion test reactor. Phys. Fluids B 5, 2554.

Nunes, I., Balboa, I., Baruzzo, M., Challis, C., Drewelow, P., Frassinetti, L., Frigione, D., Garcia, J., Hobirk, J., Joffrin, E., Lomas, P. J., Lowry, C., Rimini, F., Sips, A. C. C., WIESEN, S. \& JET EFDA contributors 2015 Compatibility of high performance operation with JET ILW. Nucl. Fusion (submitted).

Park, H. K., Luhmann, N. C. Jr., Donné, A. J. H., Classen, I. G. J., Domier, C. W., Mazzucato, E., Munsat, T., van De Pol, M. J. \& XIA, Z. 2006 Observation of highfield-side crash and heat transfer during sawtooth oscillation in magnetically confined plasmas. Phys. Rev. Lett. 96, 195003.

Pinches, S. D., Appel, L. C., Candy, J., Sharapov, S. E., Berk, H. L., Borba, D., Breizman, B. N., Hender, T. C., Hopcraft, K. I., Huysmans, G. T. A. \& Kerner, W. 1998 The HAGIS self-consistent nonlinear wave-particle interaction model. Comput. Phys. Commun. 111, 133.

Porcelli, F. 1991 Fast particle stabilisation. Plasma Phys. Control. Fusion 33, 1601.

Porcelli, F., Boucher, D. \& Rosenbluth, M. 1996 Model for the sawtooth period and amplitude. Plasma Phys. Control. Fusion 38, 2163.

Porcelli, F., Stankiewicz, R., Kerner, W. \& Berk, H. 1994 Solution of the drift-kinetic equation for global plasma modes and finite particle orbit widths. Phys. Plasmas 1, 470.

Romanelli, F. 2015 Overview of the JET results. Nucl. Fusion 55, 104001. 
Sauter, O., Westerhof, E., Mayoral, M. L., Alper, B., Belo, P. A., Buttery, R. J., Gondhalekar, A., Hellsten, T., Hender, T. C., Howell, D. F., Johnson, T., Lamalle, P., Mantsinen, M. J., Milani, F., Nave, M. F. F., Nguyen, F., Pecquet, A. L., Pinches, S. D., Podda, S. \& RAPP, J. 2002 Control of neoclassical tearing modes by sawtooth control. Phys. Rev. Lett. 88, 105001.

ShimadA, M. 2007 ITER physics basis. Nucl. Fusion 47, S1.

ZoHM, H. 2015 Recent ASDEX Upgrade research in support of ITER and DEMO. Nucl. Fusion 55, 104010.

ZuCCA, C. 2008 In Joint Varenna-Lausanne Theory Conference, vol. 1069, p. 361. AIP.

\section{Culham Prize Winner 2010}

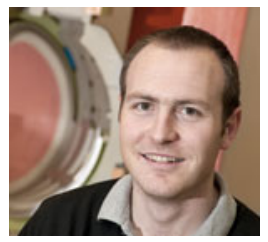

Ian Chapman received his first degree in Mathematics and Physics from Durham University in 2004 and joined Culham Centre for Fusion Energy (CCFE) as a graduate recruit. His $\mathrm{PhD}$ was awarded by Imperial College in 2008. His thesis title was 'Modelling the stability of the $n=1$ internal kink mode in tokamak plasmas' for which he was awarded the Culham Thesis Prize. His primary research interests are in understanding and controlling macroscopic instabilities in fusion plasmas. His work is characterised by a close coupling of experimental data with numerical modelling to explicate the underlying plasma physics and, by so doing, develop techniques for plasma control. His research has been recognised with a number of international awards, including the European Physical Society Early Career Prize in 2014, the Institute of Physics Paterson Medal (Best Early Career Physicist in Applied Physics) in 2013, the IUPAP Plasma Physics Young Scientist Prize in 2012 and the Cavendish Medal for Best Early-Career UK Physicist awarded by SET for Britain in 2011. He was made a Fellow of the Institute of Physics in 2013 and became a visiting Professor at Durham University in 2015. Dr Chapman became the Head of Tokamak Science at CCFE in 2014. He now leads a team of 100 scientists in both experimental plasma physics, primarily on MAST and JET but also collaborating worldwide on other fusion facilities, and theory and modelling research. 